\title{
The dependence of nonadiabatic couplings on the origin of electron coordinates
}

\section{Citation}

Belyaev, Andrey K., Alexander Dalgarno, and Ronald McCarroll. 2002. "The Dependence of Nonadiabatic Couplings on the Origin of Electron Coordinates." The Journal of Chemical Physics 116 (13) (April): 5395-5400. doi:10.1063/1.1457443.

\section{Published Version}

doi:dx.doi.org/10.1063/1.1457443

\section{Permanent link}

http://nrs.harvard.edu/urn-3:HUL.InstRepos:30403735

\section{Terms of Use}

This article was downloaded from Harvard University's DASH repository, and is made available under the terms and conditions applicable to Other Posted Material, as set forth at http:// nrs.harvard.edu/urn-3:HUL.InstRepos:dash.current.terms-of-use\#LAA

\section{Share Your Story}

The Harvard community has made this article openly available.

Please share how this access benefits you. Submit a story.

\section{Accessibility}




\title{
The dependence of nonadiabatic couplings on the origin of electron coordinates
}

\author{
Andrey K. Belyaev \\ Institute for Theoretical Atomic and Molecular Physics, Harvard-Smithsonian Center for Astrophysics, \\ Cambridge, Massachusetts 02138 and Department of Theoretical Physics, A. I. Herzen University \\ of Russia, 191186 St. Petersburg, Russia
}

\author{
Alexander Dalgarno \\ Institute for Theoretical Atomic and Molecular Physics, Harvard-Smithsonian Center for Astrophysics, \\ Cambridge, Massachusetts 02138 \\ Ronald McCarroll \\ Laboratoire de Dynamique des Ions, Atomes et Molecules UMR 7066 du CNRS, Universite Pierre et Marie \\ Curie, F-75252 Paris Cedex 05, France
}

(Received 10 December 2001; accepted 15 January 2001)

\begin{abstract}
It is shown both analytically and numerically for a number of examples that both radial and rotational nonadiabatic couplings within the standard adiabatic approach depend on the origin of the light-particle coordinates and the ambiguity in the nonadiabatic couplings does not lead to ambiguity in the coupled channel equations. The examples considered are the $\mathrm{nH}, \mathrm{nD}, \mathrm{np} \mu^{-}$ quasimolecules, for which the nonadiabatic couplings can be calculated analytically, and the $\mathrm{HeH}$ molecule, for which $a b$ initio calculations are carried out. Analytical formulas for couplings calculated with the shifted origin are derived. The coupled equations take their simplest form in Jacobi coordinates for which many nonadiabatic couplings are nonzero, even for such noninteracting systems as $\mathrm{nH}, \mathrm{nD}$, and $\mathrm{np} \mu^{-}$. These couplings are a fundamental feature of the adiabatic approach. (C) 2002 American Institute of Physics. [DOI: 10.1063/1.1457443]
\end{abstract}

\section{INTRODUCTION}

The standard adiabatic approach is the most widely used in the theoretical treatment of elementary atomic and molecular processes. It, and many others, is based on a fundamental simplification, the Born-Oppenheimer separation of slow (heavy) and fast (light) subsystems. ${ }^{1}$ Both elastic and inelastic processes can be treated. The total wave function of the system is expanded in a basis set of electronic wave functions obtained in the fixed-nuclei approximation, and the theoretical study is usually made up of two steps (see, for example, Refs. 2-5): (i) Calculations of fixed-nuclei potential energies and nonadiabatic couplings, and (ii) an appropriate treatment of the nuclear motion based on the data calculated in the first step. The approach is applicable to atomic and molecular collision processes including chemical reactions. It has been proven, both analytically and numerically, that the adiabatic potential energies are independent of the choice of origin of the coordinate system of the electrons. It is of course possible to move the origin of the entire coordinate system to any point in space without changing the value of a given nonadiabatic coupling matrix element, as confirmed by explicit quantum chemical calculations. ${ }^{6}$ However it has been pointed out ${ }^{3,7-13}$ that in contrast to potentials, nonadiabatic coupling matrix elements have different values for different electron coordinate origins. The ambiguity in the couplings seems to lead to a corresponding ambiguity in the nuclear motion, and this is often regarded as a conceptual shortcoming. ${ }^{4}$
As nonadiabatic couplings are the essence of the treatment of elementary processes, it is important to clarify how nonadiabatic matrix elements depend on the origin of the coordinate systems, and then to determine which coordinates are important for calculation of the couplings. This is the goal of the present paper.

\section{COUPLED EQUATIONS AND NONADIABATIC COUPLINGS IN JACOBI COORDINATES}

For the sake of simplicity we consider the case of two heavy particles $A$ and $B$ (nuclei) with masses $M_{A}$ and $M_{B}$ and one light particle $e$ (typically an electron) with mass $m_{e}$. The many-particle kinetic-energy operator has its simplest form in Jacobi coordinates. For three particles there exist three sets of Jacobi coordinates, but in a fixed-nuclei treatment there remains only the set of Jacobi coordinates depicted in Fig. 1(a), for which the vector $\mathbf{R}$ connects the heavy particles, and the vector $\mathbf{r}$ of the light particle is measured from the center of nuclear mass (CNM). After separation of the kinetic-energy operator of the center of mass of the total system, the Hamiltonian for the entire system is

$$
H=-\frac{\hbar^{2}}{2 M} \frac{\partial^{2}}{\partial \mathbf{R}^{2}}-\frac{\hbar^{2}}{2 m} \frac{\partial^{2}}{\partial \mathbf{r}^{2}}+H_{\mathrm{int}}(\mathbf{r}, \mathbf{R}),
$$

where $M=M_{A} M_{B} /\left(M_{A}+M_{B}\right)$ and $m=m_{e}\left(M_{A}+M_{B}\right) /\left(m_{e}\right.$ 
(a)

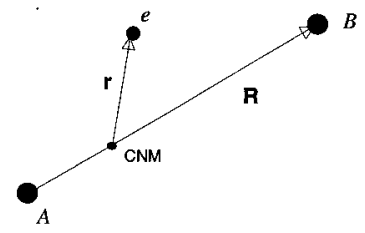

(b)

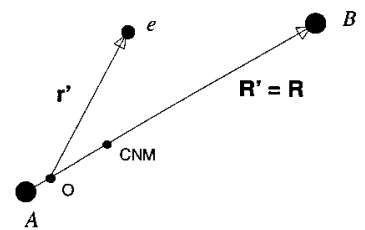

FIG. 1. (a) The Jacobi coordinates for the three particle system: $A$ and $B$ are the heavy particles, $e$ denotes the light particle, $\mathbf{r}$ is measured from the center of mass of $A$ and $B$. (b) An alternative coordinate system where $\mathbf{r}^{\prime}$ is measured from a point $O$ shifted from the center of nuclear mass.

$+M_{A}+M_{B}$ ) are the reduced masses, and the operator $H_{\text {int }}(\mathbf{r}, \mathbf{R})$ contains all the interactions. In the fixed-nuclei limit we obtain the electronic Hamiltonian

$$
H_{e}=-\frac{\hbar^{2}}{2 m} \frac{\partial^{2}}{\partial \mathbf{r}^{2}}+H_{\mathrm{int}}
$$

whose eigenfunctions are the adiabatic electronic basic functions $\phi_{j}(\mathbf{r}, \mathbf{R})$ and whose eigenvalues are the adiabatic potential energies $V_{j}(\mathbf{R})$,

$$
H_{e} \phi_{j}(\mathbf{r}, \mathbf{R})=V_{j}(\mathbf{R}) \phi_{j}(\mathbf{r}, \mathbf{R}) .
$$

A unitary transformation to diabatic states $\left|\phi_{j}^{d}(\mathbf{r}, \mathbf{R})\right\rangle$ may be performed, if desired. The wave function for the total system can be written as a sum of terms $\Psi_{M_{J}}^{J}(\mathbf{r}, \mathbf{R})$, each of which is characterized by good quantum numbers like the total angular momentum quantum numbers $J$ and $M_{J}\left(M_{J} \geqslant 0\right)$. The total scattering wave function is expanded as

$$
\Psi_{M_{J}}^{J}(\mathbf{r}, \mathbf{R})=\sum_{j} G_{j}(\mathbf{R}) \phi_{j}(\mathbf{r}, \mathbf{R}),
$$

where the functions $G_{j}(\mathbf{R})$ describe the nuclear motion. Substituting the expansion (4) into the stationary Schrödinger equation $(H-E) \Psi=0, E$ being the total energy, we get a set of coupled channel equations for the functions $G_{j}$ in the adiabatic representation,

$$
\begin{gathered}
{\left[-\frac{\hbar^{2}}{2 M} \frac{d^{2}}{d \mathbf{R}^{2}}+V_{j}(\mathbf{R})-E\right] G_{j}} \\
=\frac{\hbar^{2}}{M} \sum_{k \neq j}\left\langle\phi_{j}\left|\frac{\partial}{\partial \mathbf{R}}\right| \phi_{k}\right\rangle \frac{d G_{k}}{d \mathbf{R}} \\
+\frac{\hbar^{2}}{2 M} \sum_{k}\left\langle\phi_{j}\left|\frac{\partial^{2}}{\partial \mathbf{R}^{2}}\right| \phi_{k}\right\rangle G_{k} .
\end{gathered}
$$

The right-hand side of Eq. (5) is responsible for nonadiabatic transitions between molecular states. In a diabatic representation extra terms $\Sigma_{k \neq j}\left\langle\phi_{j}^{d}\left|H_{e}\right| \phi_{k}^{d}\right\rangle G_{k}$ may appear on the right-hand side, but the couplings $\left\langle\phi_{j}^{d}|\partial / \partial \mathbf{R}| \phi_{k}^{d}\right\rangle$ and $\left\langle\phi_{j}^{d}\left|\partial^{2} / \partial \mathbf{R}^{2}\right| \phi_{k}^{d}\right\rangle$ will disappear, if a proper transformation is used. The nonadiabatic couplings (mainly the first derivatives, but the second derivatives are needed to conserve the current) based on the adiabatic electronic wave functions are important for nonadiabatic transitions in an adiabatic representation or for a proper unitary transformation to a suitable diabatic representation.

For practical applications it is convenient to select wave functions, $F_{j \Lambda}(R)$, where $\Lambda \geqslant 0$ is the absolute value of the projection quantum number for the electronic orbital angular momentum, for the description of the radial motion of the nuclei. ${ }^{13}$ Then,

$$
\Psi_{M_{J}}^{J}(\mathbf{r}, \mathbf{R})=\sum_{j \Lambda} \frac{F_{j \Lambda}(R)}{R} I_{j \Lambda}(\mathbf{r}, \mathbf{R}),
$$

where the functions $I_{j \Lambda}$ represent the electronic motion and the angular part of the heavy particle motion. The radial wave functions are found to obey the following system of coupled equations in the adiabatic representation ${ }^{13}$

$$
\begin{aligned}
& {\left[-\frac{\hbar^{2}}{2 M} \frac{d^{2}}{d R^{2}}+V_{j \Lambda}(R)+\frac{\hbar^{2}}{2 M R^{2}}\left[J(J+1)-\Lambda^{2}\right]-E\right] F_{j \Lambda}} \\
& =\frac{\hbar^{2}}{M} \sum_{k \neq j}\left\langle\psi_{j \Lambda}\left|\frac{\partial}{\partial R}\right| \psi_{k \Lambda}\right\rangle \frac{d F_{k \Lambda}}{d R}+\frac{\hbar^{2}}{2 M} \sum_{k}\left\langle\psi_{j \Lambda}\left|\frac{\partial^{2}}{\partial R^{2}}\right| \psi_{k \Lambda}\right\rangle F_{k \Lambda} \\
& -\frac{\hbar}{M R^{2}} \sum_{k} \sqrt{(J+\Lambda+1)(J-\Lambda)}\left\langle\psi_{j \Lambda}\left|-i L_{y}\right| \psi_{k \Lambda+1}\right\rangle F_{k \Lambda+1}
\end{aligned}
$$$$
-\frac{\hbar}{M R^{2}} \sum_{k} \sqrt{(J-\Lambda+1)(J+\Lambda)}\left\langle\psi_{j \Lambda}\left|i L_{y}\right| \psi_{k \Lambda-1}\right\rangle F_{k \Lambda-1}-\frac{1}{2 M} \sum_{k}\left\langle\psi_{j \Lambda}\left|L_{x}^{2}+L_{y}^{2}\right| \psi_{k \Lambda}\right\rangle F_{k \Lambda},
$$ 
where $x, y$, and $z$ are the body-fixed components of $\mathbf{r}$, the $z$ and $\mathbf{R}$ directions coincide, and where $L_{x}$ and $L_{y}$ are the $x$ and $y$ components of the electronic angular momentum operator.

In the asymptotic $R \rightarrow \infty$ region, the adiabatic electronic basis functions become atomic eigenfunctions, and it may be shown ${ }^{12,13}$ that

$$
\left\langle\psi_{j \Lambda}\left|\frac{\partial}{\partial R}\right| \psi_{k \Lambda}\right\rangle=-\gamma_{k \Lambda} \frac{m}{\hbar^{2}}\left(V_{k \Lambda}-V_{j \Lambda}\right)\left\langle\psi_{j \Lambda}\left|z^{a t}\right| \psi_{k \Lambda}\right\rangle_{\infty}
$$

and

$$
\begin{aligned}
& \left\langle\psi_{j \Lambda}\left|i L_{y}\right| \psi_{k \Lambda \pm 1}\right\rangle \\
& =\gamma_{k \Lambda \pm 1} \frac{m}{\hbar} R\left(V_{k \Lambda \pm 1}-V_{j \Lambda}\right)\left\langle\psi_{j \Lambda}\left|x^{a t}\right| \psi_{k \Lambda \pm 1}\right\rangle_{\infty} \\
& \quad+\left\langle\psi_{j \Lambda}\left|i L_{y}^{a t}\right| \psi_{k \Lambda \pm 1}\right\rangle_{\infty},
\end{aligned}
$$

where the scalar factor $\gamma_{k \Lambda}$ is given by

$$
\gamma_{k \Lambda}=\left\{\begin{array}{c}
\gamma_{A}=-\frac{M_{B}}{M_{A}+M_{B}} \\
\quad \text { for an electron traveling with } A, \\
\gamma_{B}=+\frac{M_{A}}{M_{A}+M_{B}} \\
\quad \text { for an electron traveling with } B .
\end{array}\right.
$$

The terms on the right-hand sides in Eqs. (8) and (9) refer to atoms, e.g., $z^{a t}$ is the projection of the electron radius-vector measured from a specific nucleus onto the molecular axis. It is seen from Eqs. (7) and (9) that although the asymptotic rotational couplings increase linearly with $R$, they do not cause a problem at infinity because they are divided by $R^{2}$ in the coupled equations. In contrast, the radial couplings do not vanish, if the states are non-degenerate and are connected by a dipole transition. $3,5,12,13$

Thus, the coupled channel equations take their the simplest form (7) with the Jacobi coordinates $\mathbf{r}$ and $\mathbf{R}$, and the transitions between molecular states are driven by the radial and the rotational nonadiabatic coupling matrix elements, which should be calculated in the fixed-nuclei coordinate system with an origin at the center of nuclear mass for the electron coordinates.

\section{COUPLED EQUATIONS AND NONADIABATIC COUPLINGS IN ALTERNATIVE COORDINATES}

It may be convenient to locate the origin of the electron coordinate system at the position of one of the nuclei, a choice that avoids nonzero nonadiabatic couplings in the asymptotic region. The corresponding primed coordinates $\mathbf{r}^{\prime}$ and $\mathbf{R}^{\prime}$ are shown in Fig. 1(b). The vector $\mathbf{R}$ is unchanged. The vector $\mathbf{r}^{\prime}$ is measured from a point $O$ on the internuclear axis defined by the parameter $\gamma$ according to

$$
\mathbf{R}_{O}=\mathbf{R}_{\mathrm{CNM}}+\gamma \mathbf{R} \text {. }
$$

The parameter $\gamma$ is closely related to the parameter $\eta$ introduced in Ref. 7: $\gamma=M_{A} /\left(M_{A}+M_{B}\right)-\eta$, but $\gamma$ gives the position of $O$ with respect to the center of nuclear mass $\mathbf{R}_{\mathrm{CNM}}$, and the parameter $\eta$ does so with respect to one of the nuclei. If the origin of $\mathbf{r}^{\prime}$ is located at nucleus $A$ or $B$, then $\gamma=\gamma_{A}$ or $\gamma=\gamma_{B}$ of Eq. (10), respectively.

It is easy to show ${ }^{7,8,12}$ that

$$
\left\langle\phi_{j}\left(\mathbf{r}^{\prime}, \mathbf{R}\right)\left|\frac{\partial}{\partial \mathbf{R}}\right| \phi_{k}\left(\mathbf{r}^{\prime}, \mathbf{R}\right)\right\rangle=\left\langle\phi_{j}(\mathbf{r}, \mathbf{R})\left|\frac{\partial}{\partial \mathbf{R}}\right| \phi_{k}(\mathbf{r}, \mathbf{R})\right\rangle
$$

$$
+\gamma\left\langle\phi_{j}\left(\mathbf{r}^{\prime}, \mathbf{R}\right)\left|\frac{\partial}{\partial \mathbf{r}^{\prime}}\right| \phi_{k}\left(\mathbf{r}^{\prime}, \mathbf{R}\right)\right\rangle
$$

Using the commutation relation $\left(m / \hbar^{2}\right)\left[\mathbf{r}^{\prime}, H_{e}\right]=\partial / \partial \mathbf{r}^{\prime}$, we can write the nonadiabatic coupling matrix elements, evaluated in the system with electron coordinates referred to an origin at $O$, in terms of the adiabatic potentials, the dipole moment matrix elements, and the nonadiabatic couplings, calculated in the system of electron coordinates with origin at the center of nuclear mass, ${ }^{8,12}$ in the form

$$
\begin{gathered}
\left\langle\phi_{j}\left(\mathbf{r}^{\prime}, \mathbf{R}\right)\left|\frac{\partial}{\partial \mathbf{R}}\right| \phi_{k}\left(\mathbf{r}^{\prime}, \mathbf{R}\right)\right\rangle=\left\langle\phi_{j}(\mathbf{r}, \mathbf{R})\left|\frac{\partial}{\partial \mathbf{R}}\right| \phi_{k}(\mathbf{r}, \mathbf{R})\right\rangle \\
-\gamma\left[V_{j}(R)-V_{k}(R)\right]\left\langle\phi_{j}\left(\mathbf{r}^{\prime}, \mathbf{R}\right)\left|\mathbf{r}^{\prime}\right| \phi_{k}\left(\mathbf{r}^{\prime}, \mathbf{R}\right)\right\rangle .
\end{gathered}
$$

The nonadiabatic coupling matrix elements are different for nondegenerate states in the Jacobi and in the primed coordinates if the dipole moment matrix element is nonzero. The matrix elements of $\partial / \partial \mathbf{R}$ will change their values when another choice is made for the origin of the electron coordinates. The differentiations in Eq. (1) as well as in the matrix elements are partial derivatives, and they are altered when another set of coordinates is used.

That nonadiabatic couplings have different values for different origins of the electron coordinates has been used to claim that the coupled equations are ambiguous. ${ }^{4}$ However, it is not sufficient simply to substitute the nonadiabatic matrix elements of $\partial / \partial \mathbf{R}$, and corresponding matrix elements of the second derivative, calculated in the new coordinates, in the coupled equations (5), because the Hamiltonian of the total system is different in different coordinates. In the primed coordinates the Hamiltonian has the form

$$
\begin{aligned}
H= & -\frac{\hbar^{2}}{2 M} \frac{\partial^{2}}{\partial \mathbf{R}^{2}}-\left(\frac{\hbar^{2}}{2 m}+\gamma^{2} \frac{\hbar^{2}}{2 M}\right) \frac{\partial^{2}}{\partial \mathbf{r}^{\prime 2}} \\
& +\gamma \frac{\hbar^{2}}{M} \frac{\partial^{2}}{\partial \mathbf{R} \partial \mathbf{r}^{\prime}}+H_{\mathrm{int}} .
\end{aligned}
$$

Substitution of the expansion of the total wave function in the form of Eq. (4) in the primed coordinates leads to the following coupled channel equations for the functions $G_{j}(\mathbf{R})$ : 


$$
\begin{aligned}
& {\left[-\frac{\hbar^{2}}{2 M} \frac{d^{2}}{d \mathbf{R}^{2}}+V_{j}(\mathbf{R})-E\right] G_{j}=\frac{\hbar^{2}}{M} \sum_{k \neq j}\left[\left\langle\phi_{j}\left(\mathbf{r}^{\prime}, \mathbf{R}\right)\left|\frac{\partial}{\partial \mathbf{R}}\right| \phi_{k}\left(\mathbf{r}^{\prime}, \mathbf{R}\right)\right\rangle-\gamma\left\langle\phi_{j}\left(\mathbf{r}^{\prime}, \mathbf{R}\right)\left|\frac{\partial}{\partial \mathbf{r}^{\prime}}\right| \phi_{k}\left(\mathbf{r}^{\prime}, \mathbf{R}\right)\right\rangle\right] \frac{d G_{k}}{d \mathbf{R}}} \\
& \quad+\frac{\hbar^{2}}{2 M} \sum_{k}\left[\left\langle\phi\left(\mathbf{r}^{\prime}, \mathbf{R}\right) j\left|\frac{\partial^{2}}{\partial \mathbf{R}^{2}}\right| \phi_{k}\left(\mathbf{r}^{\prime}, \mathbf{R}\right)\right\rangle-2 \gamma\left\langle\phi_{j}\left(\mathbf{r}^{\prime}, \mathbf{R}\right)\left|\frac{\partial^{2}}{\partial \mathbf{R} \partial \mathbf{r}^{\prime}}\right| \phi_{k}\left(\mathbf{r}^{\prime}, \mathbf{R}\right)\right\rangle+\gamma^{2}\left\langle\phi_{j}\left(\mathbf{r}^{\prime}, \mathbf{R}\right)\left|\frac{\partial^{2}}{\partial \mathbf{r}^{\prime 2}}\right| \phi_{k}\left(\mathbf{r}^{\prime}, \mathbf{R}\right)\right\rangle\right] G_{k} \cdot
\end{aligned}
$$

Equations (15) differ from the coupled channel Eqs. (5) in two ways: (i) the coupling matrix elements are calculated with the primed electron coordinates, and (ii) there appear a number of new terms. However, a detailed comparison shows that the corrections cancel each other completely. Thus, substitution of the matrix elements of $\partial / \partial \mathbf{R}$ calculated in the primed coordinate, Eq. (12), into the coupled equations (15) transfers the first sum of the right-hand side of Eq. (15) into the first sum of the right hand side of Eq. (5). The same sort of compensation occurs for the matrix elements of double differentiation in the coupled equations. The coupled equations obtained with the Jacobi and with the primed coordinates are identical, a conclusion in agreement with the results of Refs. 12, 14, and 15 .

It follows that placing the origin of the electron coordinates at a nucleus does not avoid the problems with nonzero asymptotic couplings, even if both the initial and final state electronic wave functions are asymptotically centered on the same nucleus, as in direct excitation processes. It is seen from Eq. (15) that even if all the nonadiabatic matrix elements of $\partial / \partial R$ and $\partial^{2} / \partial R^{2}$ calculated in the primed coordinate with the origin at one of the nuclei are zero at $R \rightarrow \infty$, nevertheless, in the coupled equations there remain nonzero terms related to dipole moments, which cause nonadiabatic transitions between molecular states. This is a fundamental feature of the adiabatic approach for all scattering processes including chemical reactions. We set aside the problem of representing correctly the asymptotic states on an adiabatic basis set. A commonly used remedy involves a modification of the Born-Oppenheimer basis either by the introduction of electron translation factors in a semiclassical description of the dynamics, or of appropriate reaction coordinates, or of an additional unitary transformation both in a quantum description. A detailed discussion of this and other related points is outside the scope of present paper (see, e.g., Refs. 3, 5, 12, 13, and 16-20, and references therein).

The nonadiabatic matrix elements, which appear in the coupled equations (7), vary with the change of the origin of the electron coordinates as follows:

$$
\begin{aligned}
& \left\langle\psi_{j \Lambda}\left|\frac{\partial}{\partial R}\right| \psi_{k \Lambda}\right\rangle_{O}=\left\langle\psi_{j \Lambda}\left|\frac{\partial}{\partial R}\right| \psi_{k \Lambda}\right\rangle_{\mathrm{CNM}} \\
& -\gamma \frac{m}{\hbar^{2}}\left[V_{j \Lambda}(R)-V_{k \Lambda}(R)\right]\left\langle\psi_{j \Lambda}\left|z^{\prime}\right| \psi_{k \Lambda}\right\rangle, \\
& \left\langle\psi_{j \Lambda}\left|i L_{y}\right| \psi_{k \Lambda \pm 1}\right\rangle_{O}=\left\langle\psi_{j \Lambda}\left|i L_{y}\right| \psi_{k \Lambda \pm 1}\right\rangle_{\mathrm{CNM}} \\
& +\gamma \frac{m}{\hbar} R\left[V_{j \Lambda \pm 1}(R)-V_{k \Lambda}(R)\right]\left\langle\psi_{j \Lambda}\left|x^{\prime}\right| \psi_{k \Lambda \pm 1}\right\rangle,
\end{aligned}
$$

where the subscripts $O$ and CNM indicate the origin of the electron coordinates. The dipole moment matrix elements $\left\langle\psi_{j \Lambda}\left|z^{\prime}\right| \psi_{k \Lambda}\right\rangle$ and $\left\langle\psi_{j \Lambda}\left|x^{\prime}\right| \psi_{k \Lambda}\right\rangle$ are functions of $R$.

The discussion above and those in Refs. 3, 7-13 concern the dependence of the nonadiabatic couplings on the origin of the electron coordinates. The fixed-nuclei electronic Hamiltonian and its eigenfunctions have the vector $\mathbf{r}$ as a variable and depend on $\mathbf{R}$ as a parameter (or on a set of nuclear coordinates in a multidimensional case as for a polyatomic molecule), with no additional dependence on the center of nuclear mass. As a result, the adiabatic electronic wave functions in the fixed-nuclei representation and the nonadiabatic couplings are invariant with respect to the coordinate of the center of nuclear mass. The numerical results of Ref. 6 confirm that radial nonadiabatic couplings are independent of the position of the center of nuclear mass, as they must be.

Thus, nonadiabatic matrix elements on their own are not observable physical quantities and should be considered in conjunction with the dynamics of the whole system. The dependence of the nonadiabatic couplings on the origin of electron coordinates does not lead to an ambiguity in the coupled channel equations. If a system of electron coordinates with the origin shifted from the center of nuclear mass is used, the coupled equations become more complicated than those obtained with Jacobi coordinates, for which the equations have the simplest form, but the complete solutions of these sets of coupled equations must coincide.

\section{ILLUSTRATIVE EXAMPLES}

\section{A. $\mathrm{nH}$ and $\mathrm{nD}$ quasimolecules}

We consider the npe ${ }^{-}$-system $\left(A=\mathrm{p}, B=\mathrm{n}, e=e^{-}\right)$as a model for which all calculations can be performed analytically.

The electronic adiabatic molecular basis wave functions are the hydrogen atomic wave functions $\psi_{j}(\mathbf{r}, R)=\psi_{n l \tilde{m}}\left(\mathbf{r}_{\mathbf{p}}^{\prime}\right)$ $=R_{n l}\left(r_{p}^{\prime}\right) Y_{l \tilde{m}}\left(\mathbf{r}_{\mathbf{p}}^{\prime} / r_{p}^{\prime}\right)$, and independent on $R$, if the electron is measured from the proton, but dependent on $R$, if the electron is measured from the center of nuclear mass or from other points. The radial nonadiabatic couplings are equal to zero if the electron coordinates have the origin at the proton, but are nonzero between nondegenerate states with nonzero dipole moment if the electron is measured from another origin. They are given by 


$$
\begin{aligned}
& \left\langle\psi_{n^{\prime} l \tilde{m}}\left|\frac{\partial}{\partial R}\right| \psi_{n l \pm 1} \tilde{m}\right\rangle_{O}^{\mathrm{nH}} \\
& \quad=\left(\gamma-\gamma_{A}\right) \frac{m^{2} e^{4}}{2 \hbar^{4}}\left(\frac{1}{n^{\prime 2}}-\frac{1}{n^{2}}\right)\left\langle\psi_{n^{\prime} l \tilde{m}}\left|z^{\prime}\right| \psi_{n l \pm 1} \tilde{m}\right\rangle,
\end{aligned}
$$

where $\gamma_{A}=-M_{n} /\left(M_{p}+M_{n}\right)$ is close to -0.5 for the present case. The dipole moment matrix element $\left\langle\psi_{n^{\prime} l \tilde{m}}\left|z^{\prime}\right| \psi_{n l \pm 1} \tilde{m}\right\rangle$ can be evaluated analytically, and for the Lyman series we obtain

$$
\begin{aligned}
& \left\langle 1 s \Sigma\left|\frac{\partial}{\partial R}\right| n p \Sigma\right\rangle_{O}^{\mathrm{nH}} \\
& \quad=\left(\gamma-\gamma_{A}\right) 2^{3} \sqrt{\frac{n^{7}(n-1)^{2 n-5}}{3(n+1)^{2 n+5}}}\left(1-\frac{1}{n^{2}}\right) \frac{m e^{2}}{\hbar^{2}} .
\end{aligned}
$$

The radial coupling between the ground $1 s \Sigma$ state and the excited $2 p \Sigma$ state, calculated with respect to the center of nuclear mass $(\gamma=0)$, is $\langle 1 s \Sigma|\partial / \partial R| 2 p \Sigma\rangle_{\mathrm{CNM}}^{\mathrm{nH}}=0.140$ a.u., but the same matrix element equals $\langle 1 s \Sigma|\partial / \partial R| 2 p \Sigma\rangle_{p}^{\mathrm{nH}}$ $=0$ with respect to the proton $\left(\gamma=\gamma_{A}\right)$, and $\langle 1 s \Sigma|\partial / \partial R| 2 p \Sigma\rangle_{n}^{\mathrm{nH}}=0.280$ a.u. with respect to the neutron $\left[\gamma=M_{p} /\left(M_{p}+M_{n}\right)\right]$. If the origin of the electron coordinates varies along the internuclear axis, the radial nonadiabatic coupling $\langle 1 s \Sigma|\partial / \partial R| 2 p \Sigma\rangle_{O}^{\mathrm{nH}}$ is a linear function of $\gamma$ or $\eta$, as shown in Fig. 2. The result of the test calculations of Ref. 6 for the radial nonadiabatic coupling for $\mathrm{HCl}$ also yields a linear dependence of the couplings on the parameter $\gamma$, although it was interpreted differently. (The opposite sign of $\gamma$ in Ref. 6 does not change the conclusion.)

The nonadiabatic rotational coupling matrix elements can be also evaluated analytically for the npe ${ }^{-}$system, but in contrast to the radial couplings, they are $R$-dependent, as seen from Eq. (17). For the Lyman series they are given by

$$
\begin{aligned}
& \left\langle 1 s \Sigma\left|i L_{y}\right| n p \Pi\right\rangle_{O}^{\mathrm{nH}} \\
& \quad=R\left(\gamma-\gamma_{A}\right) 2^{3} \sqrt{\frac{n^{7}(n-1)^{2 n-5}}{3(n+1)^{2 n+5}}}\left(1-\frac{1}{n^{2}}\right) \frac{m e^{2}}{\hbar} .
\end{aligned}
$$

Putting the origin of the electron coordinates at the positions of the proton, the center of nuclear mass, and the neutron, respectively, we obtain for the rotational nonadiabatic couplings between the $1 s \Sigma$ and $2 p \Pi$ states equal $0,0.140 R$ a.u., and $0.280 R$ a.u., as shown in Fig. 3 (the dotted, the solid, and the dotted-dashed lines, respectively). The rotational coupling calculated with respect to the center of nuclear mass increases to infinity with $R$ in the asymptotic region.

The values of nonadiabatic couplings are different for isotopic species, because the position of the center of nuclear mass on the molecular axis changes, but the general conclusion holds: nonadiabatic couplings depend on the origin of the electron coordinates. The formulas above are valid for nd $e^{-}$, with the proton mass replaced by the deuteron mass. Thus, $\langle 1 s \Sigma|\partial / \partial R| 2 p \Sigma\rangle_{\mathrm{CNM}}^{\mathrm{nD}}=0.093$ a.u. and $\left\langle 1 s \Sigma\left|i L_{y}\right| 2 p \Pi\right\rangle_{\mathrm{CNM}}^{\mathrm{nD}}=0.093 R$ a.u., when the electron is measured from the center of nuclear mass (the dashed lines in Figs. 2 and 3).

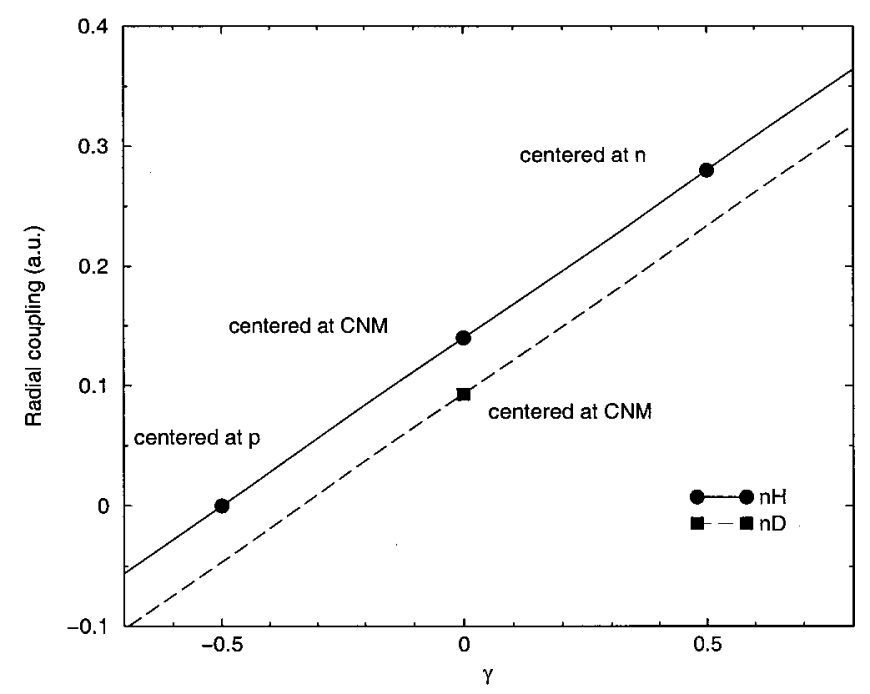

FIG. 2. The radial nonadiabatic couplings $\langle 1 s \Sigma|\partial / \partial R| 2 p \Sigma\rangle$ for the $\mathrm{nH}$ (the solid line) and the $\mathrm{nD}$ (the dashed line) systems as functions of the parameter $\gamma$ which specifies the position of the origin of the electron coordinates. The circles and the square are the particular values of the couplings calculated with respect to the origin at the center of nuclear mass or at one of the nuclei.

\section{B. An np $\mu^{-}$quasimolecule}

The situation changes dramatically, when the electron is replaced by a muon. The formulas remain the same, but the mass of the light particle is much larger: $m_{\mu}=206.768 m_{e}$. As a result, nonadiabatic couplings are nearly 200 times larger. For example, the radial and the rotational couplings calculated with respect to the center of nuclear mass are $\langle 1 s \Sigma|\partial / \partial R| 2 p \Sigma\rangle_{\mathrm{CNM}}^{\mathrm{np} \mu}=27.36$ a.u. and $\left\langle 1 s \Sigma\left|i L_{y}\right| 2 p \Pi\right\rangle_{\mathrm{CNM}}^{\mathrm{np} \mu}$ $=27.36 R$ a.u., respectively.

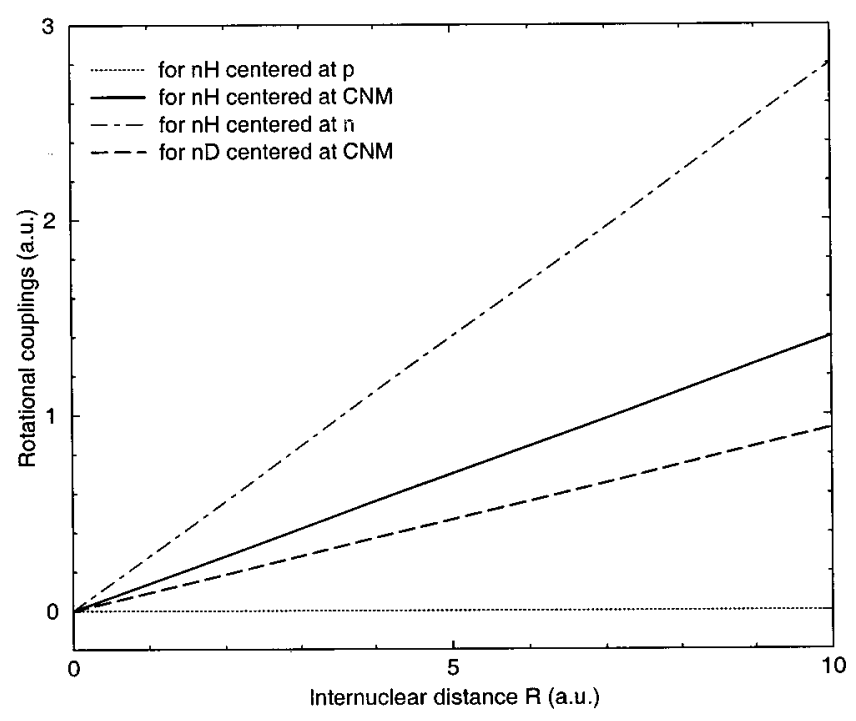

FIG. 3. The rotational coupling matrix elements $\left\langle 1 s \Sigma\left|i L_{y}\right| 2 p \Pi\right\rangle$ for the $\mathrm{nH}$ and the $\mathrm{nD}$ systems as functions of the internuclear distance $R$ calculated with different origins of the electron coordinates. 


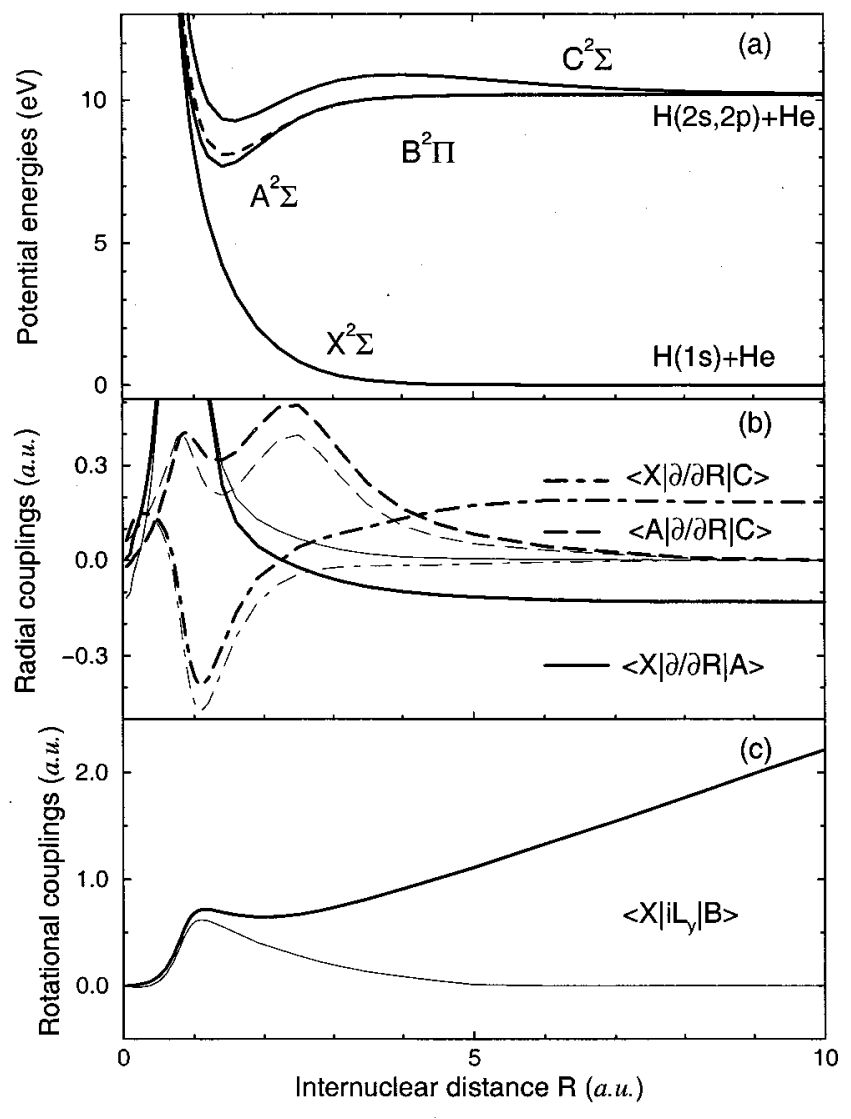

FIG. 4. The adiabatic potentials (a), the radial (b), and the rotational (c) couplings for the $\mathrm{HeH}$ system calculated with the origin of the electron coordinates at the center of nuclear mass (thick lines) and at the proton (thin lines).

\section{An HeH quasimolecule}

A many-electron system with one active electron and a number of inactive electrons may be treated by means of the approach described above. We consider the $\mathrm{HeH}$ molecule. The adiabatic potentials, the radial and the rotational nonadiabatic couplings are calculated $a b$ initio by the quantum chemical programs that includes configuration interactions. ${ }^{21-23}$ Data computed with the origin of the electron coordinates at the center of nuclear mass (thick lines) and at the position of proton (thin lines) are shown in Fig. 4. The adiabatic potentials calculated with different origins coincide. The radial nonadiabatic couplings [Fig. 4(b)] vary with the change of the origin of the electron coordinates in accordance with Eq. (16). Two couplings, $\left\langle X^{2} \Sigma|\partial / \partial R| A^{2} \Sigma\right\rangle$ and $\left\langle X^{2} \Sigma|\partial / \partial R| C^{2} \Sigma\right\rangle$, are nonzero in the asymptotic region, because of the degeneracy of the $\left|A^{2} \Sigma\right\rangle$ and $\left|C^{2} \Sigma\right\rangle$ molecular states at infinity, ${ }^{24}$ although only one coupling $\left\langle 1 s^{2} \Sigma|\partial / \partial R| 2 l^{2} \Sigma\right\rangle$ between the atomic states is nonzero. The rotational couplings also differ with different origins of the electron coordinates according to Eq. (17). The most remarkable difference is between the $\left|X^{2} \Sigma\right\rangle$ and $\left|B^{2} \Pi\right\rangle$ states, the matrix element for which is plotted in Fig. 4(c). The rotational coupling increases linearly with $R$ if the electrons are referred to the center of nuclear mass (compare with Fig. $3)$. More examples of varying the nonadiabatic coupling matrix elements can be found in Refs. 7-11.

\section{CONCLUSION}

It is shown that within the standard adiabatic approach both radial and rotational nonadiabatic coupling matrix elements depend on and only on the origin of the light-particle coordinates, and this dependence does not lead to ambiguity in the coupled channel equations, which are invariant with respect to the choice of the origin. The adiabatic basis wave functions and nonadiabatic couplings are invariant with respect to the coordinate of the center of nuclear mass. Analytical and numerical calculations for the cases $\mathrm{nH}, \mathrm{nD}$, $\mathrm{np} \mu^{-}$, and $\mathrm{HeH}$ confirm the theoretical conclusion and are in agreement with the analytical formulas for radial and rotational couplings. In particular, it is shown that certain of the nonadiabatic coupling terms for the noninteracting systems $\mathrm{nH}, \mathrm{nD}$, and $\mathrm{np} \mu^{-}$are nonzero, if calculated in the Jacobi coordinates for which the coupled channel equations take their simplest form. This is a fundamental feature of the standard adiabatic approach and it cannot be removed by shifting the origin of the coordinate systems.

\section{ACKNOWLEDGMENTS}

This work was supported by the National Science Foundation through a grant for the Institute for Theoretical Atomic and Molecular Physics at Harvard University and Smithsonian Astrophysical Observatory. A.D. was supported by the Chemical Sciences, Geosciences, and Biosciences Division of the Office of Basic Energy Sciences, Office of Science, U.S. Department of Energy.

${ }^{1}$ M. Born and J. R. Oppenheimer, Ann. Phys. (Leipzig) 84, 457 (1927).

${ }^{2}$ N. F. Mott and H. S. W. Massey, The Theory of Atomic Collisions (Clarendon, Oxford, 1933).

${ }^{3}$ A. Macias and A. Riera, Phys. Rep. 90, 299 (1982).

${ }^{4}$ B. H. Bransden and M. R. C. McDowell, Charge Exchange and the Theory of Ion-Atom Collisions (Clarendon, Oxford, 1992).

${ }^{5}$ R. McCarroll and D. S. F. Crothers, Adv. At., Mol., Opt. Phys. 32, 253 (1994).

${ }^{6}$ R. Buenker and Y. Li, J. Chem. Phys. 112, 8318 (2000).

${ }^{7}$ T. G. Heil, S. E. Butler, and A. Dalgarno, Phys. Rev. A 23, 1100 (1981).

${ }^{8}$ M. Gargaud, J. Hanssen, R. McCarroll, and P. Valiron, J. Phys. B 14, 2259 (1981).

${ }^{9}$ M. Gargaud and R. McCarroll, J. Phys. B 18, 463 (1985).

${ }^{10}$ O. Mo, A. Riera, and M. Yanez, Phys. Rev. A 31, 3977 (1985).

${ }^{11}$ B. Zygelman and A. Dalgarno, Phys. Rev. A 33, 3853 (1986).

${ }^{12}$ J. Grosser, T. Menzel, and A. K. Belyaev, Phys. Rev. A 59, 1309 (1999).

${ }^{13}$ A. K. Belyaev, D. Egorova, J. Grosser, and T. Menzel, Phys. Rev. A 64, 052701 (2001).

${ }^{14}$ B. Zygelman, D. L. Cooper, M. J. Ford, A. Dalgarno, J. Gerratt, and M. Raimondi, Phys. Rev. A 46, 3846 (1992).

${ }^{15}$ G. J. Bottrell, Nucl. Instrum. Methods Phys. Res. B 79, 173 (1993).

${ }^{16}$ D. R. Bates and R. McCarroll, Proc. R. Soc. London, Ser. A 245, 175 (1958).

${ }^{17}$ S. B. Schneidermann and A. Russek, Phys. Rev. 181, 311 (1969).

${ }^{18}$ M. H. Mittleman, Phys. Rev. 188, 221 (1969).

${ }^{19}$ J. B. Delos, Rev. Mod. Phys. 53, 287 (1981).

${ }^{20}$ J. Robert and J. Baudon, J. Phys. B 19, 171 (1986).

${ }^{21}$ G. Hirsch, P. J. Bruna, R. J. Buenker, and S. D. Peyerimhoff, Chem. Phys. 45, 335 (1980).

${ }^{22}$ P. J. Bruna and S. Peyerimhoff, Adv. Chem. Phys. 67, 1 (1987).

${ }^{23}$ P. J. Knowles and H.-J. Werner, MOLPRO quantum chemistry package (University of Birmingham, 2000).

${ }^{24}$ A. K. Belyaev, J. Grosser, and T. Menzel (to be published). 